\title{
Incremento na obtenção de oócitos em novilhas Nelore (Bos taurus indicus) tratadas com progesterona injetável e benzoato de estradiol
}

\author{
Enhancement of oocytes obtainment in Nelore heifers \\ (Bos taurus indicus) treated with progesterone injection and \\ benzoate of estradiol
}

\author{
Danilo Bacelar'; Marilu Constantino Max²; Luciana C. Padilha ${ }^{1}$; \\ Thales Ricardo R. Barreiros ${ }^{3}$; Marcelo Marcondes Seneda ${ }^{1 *}$
}

\begin{abstract}
Resumo
O objetivo deste estudo foi comparar dois protocolos pré-aspiração folicular associados com PGF2 $\alpha$. Foram utilizadas 14 novilhas da raça Nelore, não gestantes, com escore corporal de 2,5 (escala de 1 a 5). Todos os animais foram submetidos aos dois tratamentos (cross over) para controle da onda de crescimento folicular, um através da ablação de todos os folículos 3 dias antes da aspiração folicular (PAF) e outro por controle hormonal (PCH) com benzoato de estradiol e progesterona injetável. Os dados foram analisados pelo ANOVA e as diferenças significativas pelo teste de Tukey, com nível de significância de $5 \%$. O protocolo controle hormonal ( $\mathrm{PCH})$ mostrou-se vantajoso por propiciar maior média de folículos e oócitos, além de redução no número de corpos lúteos evitando também o procedimento extra de aspiração prévia para ablação dos folículos. Conclui-se que o protocolo hormonal $(\mathrm{PCH})$ apresentado mostra-se uma alternativa viável para tratamentos pré-aspiração, havendo benefícios adicionais pela ausência de corpo lúteo no momento da aspiração folicular.
\end{abstract}

Palavras-chave: Aspiração folicular, bovinos, protocolo pré-aspiração folicular

\begin{abstract}
The aim of this study was to evaluate two pre-aspiration protocols associated with PGF2 $\alpha$. Nelore heifers ( $\mathrm{n}=14$ ), not pregnant, body score of 2.5 (scale of 1 to 5) were used. All animals were submitted to two treatments (cross over) to control the follicular wave of growth, one by follicular ablation 3 days before the aspiration (PAF) and another by hormonal control with estrogen and injectable progesterone $(\mathrm{PCH})$. Data were analyzed by ANOVA and significant differences were compared by Tukey test, with significance level of 5\%. The hormonal control protocol $(\mathrm{PCH})$ provided advantages as higher average of follicles and oocytes, reduction in the number of corpus luteum and one procedure less - the follicle ablation. We concluded that the hormone control protocol ( $\mathrm{PCH})$ is a viable alternative for preaspiration treatments, with additional benefits for the absence of corpus luteum at the time of follicular aspiration.
\end{abstract}

Key words: Follicular aspiration, bovine, protocol of follicular pre-aspiration

${ }^{1}$ Laboratório de Reprodução Animal, DCV/CCA/UEL, Londrina, PR. E-mail: mseneda@uel.br

2 Laboratório de Reprodução Animal, DCV/CCA/UEL, Londrina, PR.. E-mail: marilucmax@hotmail.com.

3 Departamento de Produção Animal, UENP/FALM, Bandeirantes, PR.

Autor para correspondência 


\section{Introdução}

Nos últimos anos a produção in vitro de embriões bovinos vem se destacando em virtude dos resultados promissores obtidos na raça Nelore (Bos taurus indicus) (NONATO JÚNIOR et al., 2004). A aspiração folicular guiada por ultra-sonografia (Ovum Pick-up - OPU) permite a obtenção de oócitos uma ou duas vezes por semana sem conseqüências imediatas para o sistema genital dos bovinos (BOLS; VANDENHEEDE; VAN SOOM, 1995).

Até pouco tempo, a aspiração folicular era geralmente realizada em momentos aleatórios do ciclo estral. No entanto, a crescente necessidade de melhorar a eficiência da técnica e o acréscimo de demanda para situações menos favoráveis (animais com menor produção de oócitos) apontam para um incremento na utilização de protocolos pré-aspiração (SENEDA et al., 2006).

Naturalmente, o número de folículos disponíveis para a aspiração folicular apresenta considerável variação conforme a fase de crescimento folicular, sendo o início da onda o momento mais favorável para a recuperação, pelo maior número de folículos e pela melhor eficiência na captação dos oócitos a partir de folículos menores (SENEDA et al., 2001).

Quando a aspiração folicular é realizada independente da fase do ciclo estral, mais de $85 \%$ dos folículos ovarianos são atrésicos. Existem fortes evidências de que o estágio de desenvolvimento do folículo é importante para determinar a qualidade do oócito (CASTILHO; ASSIS; GARCIA, 2007; SIRARD et al., 1999).

Vários protocolos de superestimulação ovariana, com o uso de gonadotrofinas (FSH, PMSG ou eCG e r-bST), têm sido estudados visando aumentar a eficiência desta técnica (BOLS et al., 1998; DE ROOVER et al., 2005; PIETERSE et al., 1992; SENEDA et al., 2005). As vantagens da estimulação folicular parecem óbvias, mais folículos resultariam em mais oócitos (DE ROOVER et al., 2005) entretanto, há muitos conflitos sobre esta prática.
Apesar dos protocolos pré-aspiração promoverem aumento de tamanho dos ovários, tornando mais fácil sua manipulação (TECHAKUMPHU et al., 2004), e ainda proporcionarem maior número de folículos viáveis por aspiração, a eficiência da recuperação dos oócitos diminuiu com o uso do FSH ou BST (BOLS et al., 1998; PIETERSE et al., 1992; WALTON; CHRISTIE; STUBBINGS, 1993). Supõe-se que os folículos com diâmetros maiores produzidos por estes protocolos poderiam induzir uma menor eficiência da recuperação oocitária devido aos seguintes aspectos: maior pressão intrafolicular, fluido folicular mais viscoso e em maior quantidade, o que dificulta a aspiração dos mesmos (SENEDA et al., 2001).

Protocolos pré-aspiração também foram desenvolvidos com o objetivo de controlar a onda de crescimento folicular. Dentre as formas de controle do crescimento folicular, o tratamento com progesterona e estradiol têm sido eficazes para promover a atresia dos folículos em desenvolvimento e a emergência de uma nova onda folicular 3 a 5 dias após, devido a ação inibitória destes dois hormônios sobre o FSH (BÓ et al., 1994, 2006). Através da administração de $2 \mathrm{mg}$ de benzoato de estradiol e $50 \mathrm{mg}$ de progesterona no momento da inserção do implante de progestágeno foi possível observar a emergência de uma nova onda folicular, em média 4 dias (BARUSELLI et al., 2006; MORENO et al., 2001). Adicionalmente, somente a aspiração folicular também pode controlar o crescimento folicular, promovendo a emergência de uma nova onda folicular após 1 a 2 dias (BERFELT; LIGHTFOOT; ADAMS, 1994; CAVALIERI et al., 2001).

Embora implantes de progestágenos sejam bastante utilizados em protocolos prévios à aspiração folicular, o curto período de utilização dos mesmos permite o questionamento de sua necessidade. Um outro aspecto importante refere-se à aplicação de PGF2 $\alpha$ previamente a aspiração, visando evitar a presença de corpo lúteo no momento da aspiração folicular, facilitando desta maneira a manipulação dos ovários (SENEDA et al., 2006). 
O objetivo deste estudo foi testar um protocolo pré-aspiração folicular com uso de progesterona injetável, benzoato de estradiol e prostaglandina, quanto ao diâmetro folicular, recuperação dos oócitos e presença ou não de corpo lúteo no momento da aspiração.

\section{Material e métodos}

\section{Local e animais}

Foram utilizadas 14 novilhas da raça Nelore (Bos taurus indicus), não gestantes e com escore corporal de 2,5 (escala de 1 a 5) (LOWMAN; SCOTT; SOMERVILLE, 1976), durante o período de janeiro a fevereiro de 2006. Estes animais foram mantidos em pastagem de Brachiaria decumbens e suplementados com sal mineral contendo $9 \%$ de fósforo na mistura, no município de Bauru, Estado de São Paulo.

\section{Tratamentos}

As novilhas foram submetidas a dois tratamentos para controle da onda de crescimento folicular, com intervalos de 14 dias e 2 repetições, em um delineamento experimental cross-over, de forma que todos os animais passaram pelos dois tratamentos. Dessa maneira foram realizadas 56 sessões de aspiração folicular (OPU). No Protocolo Aspiração Folicular (PAF, $\mathrm{n}=28$ ), os animais receberam no (D0) 0,5 mg de cloprostenol sódico por via intramuscular (IM) (Sincrocio, Ourofino, Brasil) e foram submetidos à aspiração de todos os folículos visualizados na ultra-sonografia (Aloka SSD 500, transdutor endovaginal, microconvexo, $5 \mathrm{MHz}$ ). Setenta e duas horas depois (D3), momento em que é prevista a emergência de uma nova onda de crescimento folicular, foi realizada uma outra aspiração folicular para obtenção de oócitos e também se avaliou a presença de corpo lúteo (CL). No segundo tratamento, denominado Protocolo Controle Hormonal ( $\mathrm{PCH}, \mathrm{n}=28$ ) foram administrados no (D0) $2 \mathrm{mg}$ de benzoato de estradiol (Estrogin, Farmavet, São Paulo), 0,5 mg de cloprostenol sódico e $50 \mathrm{mg}$ de progesterona IM (Progesterona injetável, Syntex, Argentina). Seis dias depois (D6), foi realizada a aspiração folicular ou OPU. Os tratamentos estão ilustrados na Figura 1.

\section{Tratamento PAF $(n=28)$}

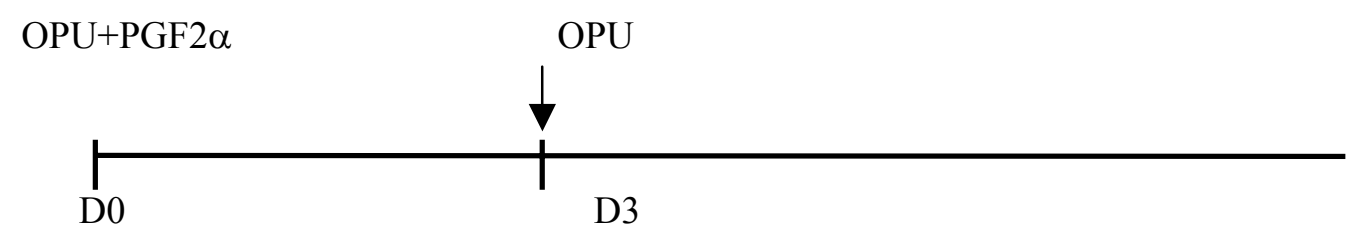

\section{Tratamento $\mathrm{PCH}(\mathrm{n}=\mathbf{2 8})$}

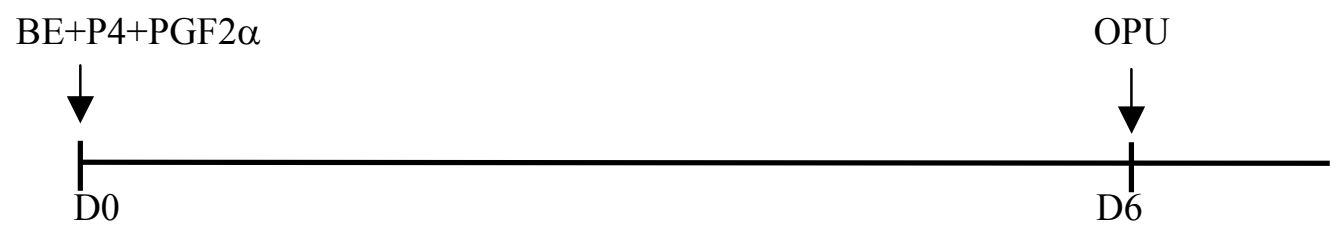

Figura 1. Representação esquemática dos tratamentos PAF (Protocolo Aspiração Folicular) e PCH (Protocolo Controle Hormonal) prévios à aspiração folicular (OPU) realizados em novilhas Nelore (Bos taurus indicus), no município de Bauru, Estado de São Paulo, de janeiro a fevereiro de 2006. (PGF2 $\alpha$ : Prostaglandina; BE: Benzoato de estradiol; P4: Progesterona). 


\section{Equipamento}

Utilizou-se ultra-som da marca Aloka, modelo SSD-500 (Japão), em tempo real, com transdutor microconvexo de $5 \mathrm{MHz}$, adaptado a uma guia para a aspiração folicular transvaginal. Para realização da punção folicular foi utilizada agulha hipodérmica descartável (40x9 mm comprimento, Becton Dickson, Brasil) conectada a um tubo cônico de 50 $\mathrm{ml}$ (Corning, USA) por uma mangueira de silicone de $0,8 \mathrm{~m}$ e diâmetro interno de $2 \mathrm{~mm}$. A aspiração foi realizada a partir de uma pressão negativa correspondente à vazão de $13 \mathrm{ml}$ de $\mathrm{H}_{2} 0 / \mathrm{min}$, gerada por uma bomba de vácuo (Rocket Medical, England). O meio utilizado para aspiração foi DPBS (Nutricell, Brasil) suplementado com $10 \%$ de soro fetal bovino (Nutricell, Brasil) e heparina (17 UI/ml).

\section{Preparação das doadoras}

Antes de cada procedimento de aspiração, as fezes eram retiradas do reto e a região perineal era higienizada com água e álcool $70 \%$. Em seguida, realizava-se a anestesia epidural com $7 \mathrm{ml}$ de lidocaína $2 \%$ para diminuir o peristaltismo e o desconforto. $\mathrm{O}$ transdutor era inserido na vagina e posicionado no fórnix, até que uma imagem satisfatória dos ovários fosse obtida.

\section{Aspiração folicular}

Os folículos de ambos os ovários foram mensurados e classificados de acordo com os seguintes diâmetros: $<5 \mathrm{~mm}$ e $>5 \mathrm{~mm}$. Em seguida, todos os folículos eram contados e subsequentemente aspirados, independentemente do seu diâmetro. Outro dado observado durante as aspirações foi quanto à presença ou não de corpo lúteo (CL). Para a obtenção dos oócitos, a agulha era pressionada contra a parede vaginal, introduzida até o ovário, e então inserida nos folículos individualmente, no mesmo momento que a bomba de aspiração era ativada. Após a aspiração de todos os folículos, era realizada a aspiração de $20 \mathrm{~mL}$ de meio para recuperar os oócitos que eventualmente se encontrassem no interior da agulha. Imediatamente após a aspiração, o fluido folicular recuperado era passado por um filtro de 70 micras (Emcom, Nutricell, Brasil) e subsequentemente lavado com PBS (Nutricell - Brasil) suplementado com $10 \%$ de soro fetal bovino (Nutricell - Brasil). Finalmente era realizada a busca e classificação dos oócitos. Todos os procedimentos foram realizados pelo mesmo Médico Veterinário.

\section{Recuperação e avaliação dos oócitos}

Imediatamente após a recuperação dos oócitos, foi realizada a classificação dos mesmos de acordo com as características das células do complexo cumulus oophorus (CCO) e do ooplasma: Grau I(GI) - Cumulus compacto presente, contendo mais de três camadas de células. Ooplasma com granulações finas e homogêneas, preenchendo o interior da zona pelúcida e de coloração marrom (ou presente mais do que três fileiras de células do cumulus); Grau II (GII)-Cumulus compacto parcialmente presente em volta do oócito ou rodeando completamente o oócito, com menos de três camadas celulares. Ooplasma, com granulações distribuídas heterogeneamente, podendo estar mais concentradas no centro e mais claras na periferia ou condensadas em um só local aparentando uma mancha escura; Grau III (GIII) - Cumulus presente, mas expandido. Ooplasma contraído, com espaço entre a membrana celular e a zona pelúcia, preenchendo irregularmente o espaço perivitelino; Degenerado, vacuolizado ou fragmentado; Desnudos - totalmente descobertos pelas células do cumulus ou parte coberto por elas; atrésico - cumulus oophorus escuro ou presença de sinais de degeneração citoplasmática (SENEDA et al., 2001). 


\section{Análise estatística}

A análise estatística foi realizada pelo programa computacional MINITAB 14 (MINITAB INC, 1992). As variáveis, inicialmente, foram submetidas ao Teste de Kolmogorov-Smirnov para verificar se os dados apresentavam ou não distribuição paramétrica. As variáveis foram submetidas à análise de variância, havendo diferença foi procedido um pós-teste de Tukey, com nível de significância de 5 $\%(\mathrm{p}=0,05)$.

\section{Resultados}

Após 56 procedimentos, foram aspirados 508 folículos, sendo 201 no grupo PAF e 307 folículos no grupo PCH (Tab. 1). Portanto, houve diferença significativa $(\mathrm{P}<0,05)$ no total de folículos aspirados entre os tratamentos. As médias de folículos e oócitos por animal no PAF e $\mathrm{PCH}$, foram, respectivamente, 7,2 / 5,4 e 11 / 7,4 ( $\mathrm{P}<0,05$; Tab. 1). Foi observada uma predominância de folículos $<5 \mathrm{~mm}$ nos dois tratamentos testados, com média por animal e por aspiração de 5,5 no PAF e 8,7 no PCH. Houve diferença significativa $(\mathrm{P}<0,05)$ na quantidade de folículos $<5 \mathrm{~mm}$, mas não entre os folículos $>5$ $\mathrm{mm}(\mathrm{P}>0,05)$ entre PAF e PCH.

Em ambos os tratamentos ocorreu predominância de oócitos de Grau III, entretanto houve diferença significativa $(\mathrm{P}<0,05)$ entre os oócitos de Grau II, com total de 34 no PAF e 67 no PCH (Tab. 2).

No momento da aspiração foi avaliada a presença ou não de corpos lúteos. Foram encontrados no total doze corpos lúteos (CL), sendo 35,7 \% (10/28) no grupo PAF e 7,1 \% (2/28) no grupo $\mathrm{PCH}$, havendo diferença estatística $(\mathrm{P}<0,05)$ (Fig. 2).

Tabela 1. Efeito dos tratamentos PAF (Protocolo Aspiração Folicular) e PCH (Protocolo Controle Hormonal) no número de folículos $<5 \mathrm{~mm}$ e $>5 \mathrm{~mm}$ e no número de oócitos aspirados, Bauru-SP, janeiro a fevereiro de 2006.

\begin{tabular}{cccccc}
\hline Tratamentos & $\mathrm{N}$ & $\begin{array}{c}\text { Folículos } \\
<5 \mathrm{~mm} \\
(\text { média } \pm \text { SEM) }\end{array}$ & $\begin{array}{c}\text { Folículos } \\
>5 \mathrm{~mm} \\
(\text { média } \pm \text { SEM) }\end{array}$ & $\begin{array}{c}\text { Total } \\
\text { Folículos } \\
(\text { média } \pm \text { SEM) }\end{array}$ & $\begin{array}{c}\text { Oócitos } \\
(\text { média } \pm \text { SEM) }\end{array}$ \\
\hline PAF & 28 & $154(5,5 \pm 2,4)^{\mathrm{Aa}}$ & $63(1,6 \pm 1,0)^{\mathrm{B}}$ & $201(7,2 \pm 2,6)^{\mathrm{a}}$ & $162(5,4 \pm 3,0)^{\mathrm{a}}$ \\
PCH & 28 & $244(8,7 \pm 3,7)^{\mathrm{Ab}}$ & $47(2,2 \pm 1,2)^{\mathrm{B}}$ & $307(11 \pm 4,5)^{\mathrm{b}}$ & $226(7,4 \pm 3,3)^{\mathrm{b}}$ \\
\hline
\end{tabular}

Resultados seguidos de diferentes letras minúsculas na mesma coluna diferem estatisticamente $(\mathrm{P}<0,05)$.

Tabela 2. Efeito dos tratamentos PAF (Protocolo Aspiração Folicular) e PCH (Protocolo Controle Hormonal) sobre a quantidade total e qualidade dos oócitos obtidos mediante aspiração folicular de Novilhas Nelore, Bauru-SP, janeiro a fevereiro de 2006.

\begin{tabular}{cccccccc}
\hline \multirow{2}{*}{ Tratamentos } & & \multicolumn{7}{c}{ Oócitos (\%) } \\
\cline { 3 - 7 } & $\mathrm{N}$ & GI & GII & GIII & Desnudo & Atrésico & Total \\
\hline PAF & 28 & $18(11,1)^{\mathrm{A}}$ & $34(20,9)^{\mathrm{Ba}}$ & $78(48,1)^{\mathrm{C}}$ & $30(18,5)^{\mathrm{A}}$ & $2(1,2)^{\mathrm{D}}$ & $162(100)^{\mathrm{c}}$ \\
PCH & 28 & $27(11,9)^{\mathrm{A}}$ & $67(29,6)^{\mathrm{Bb}}$ & $84(37,1)^{\mathrm{B}}$ & $38(16,8)^{\mathrm{D}}$ & $10(4,4)^{\mathrm{C}}$ & $226(100)^{\mathrm{d}}$ \\
\hline
\end{tabular}

Resultados seguidos de diferentes letras maiúsculas na mesma linha diferem estatisticamente $(\mathrm{P}<0,05)$; Resultados seguidos de diferentes letras minúsculas na mesma coluna diferem estatisticamente $(\mathrm{P}<0,05)$. 


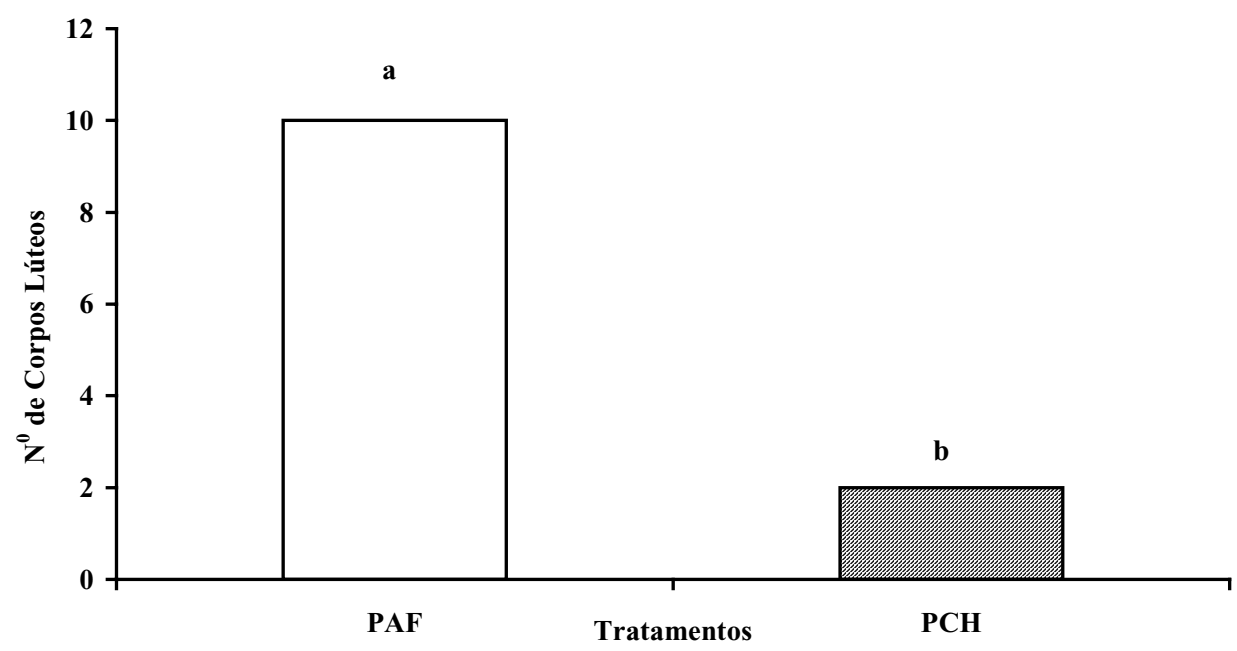

Figura 2. Efeito dos tratamentos PAF (Protocolo Aspiração Folicular) e PCH (Protocolo Controle Hormonal) na quantidade de corpos lúteos (CL) encontrados durante os procedimentos de aspiração folicular via ultra-sonografia transretal, Bauru-SP, janeiro a fevereiro de 2006. Resultados seguidos de letras diferentes diferem estatisticamente $(\mathrm{P}<0,05)$.

\section{Discussão}

No presente trabalho, foi encontrado um maior número de folículos com diâmetro $<5 \mathrm{~mm}$ em ambos os tratamentos. Isto pode ser explicado pelo fato de que a aspiração folicular foi realizada no início da onda de crescimento folicular tanto no PAF como no PCH. No PCH os folículos presentes na ocasião do tratamento regrediram através da ação conjunta da progesterona e do estrógeno e no PAF essa regressão foi realizada mecanicamente através da aspiração de todos os folículos visíveis no ovário anteriormente à aspiração folicular. Em conseqüência aos dois procedimentos, iniciouse uma nova onda de crescimento folicular aproximadamente 6 dias após o PCH e 3 dias após o PAF. Comparando somente os folículos $<5 \mathrm{~mm}$ de cada tratamento, observou-se que no $\mathrm{PCH}$ o número destes foi significativamente maior. Essa situação é muito favorável, visto que Seneda et al. (2001) mostraram uma melhor eficiência no número de oócitos recuperados a partir de folículos $<5 \mathrm{~mm}$ de diâmetro. Por outro lado, com folículos $>5 \mathrm{~mm}$, espera-se encontrar maior número de folículos atrésicos, em razão da dominância folicular que ocorre nos bovinos (GINTHER, 2000). A presença do folículo dominante também reduz significativamente a competência dos oócitos inclusosem folículos subordinados (HENDRIKSEN etal.,2004), além de interferirno número de folículos visualizados (TORRES-JÚNIOR et al., 2006). No entanto, Sendeda et al. (2001) demonstraram não haver diferença da qualidade do cumulus oophorus, entre oócitos de folículos grandes e pequenos, além de não ter sido observada influência do diâmetro do folículo sobre o desenvolvimento embrionário. Resultados similares foram observados sobre a capacidade de desenvolvimento embrionário de oócitos obtidos de folículos de 3-5 $\mathrm{mm}$ ou $>5 \mathrm{~mm}$ (BLONDIN; SIRARD, 1995). Seneda et al. (2001) encontraram grande número de folículos $>5 \mathrm{~mm}$, sendo mais fáceis de serem puncionados do que os folículos $<5 \mathrm{~mm}$. Resultados semelhantes já haviam sido mostrados previamente por Goodhand et al. (1999).

O fato de termos aspirado folículos no período inicial de crescimento também é favorável, pois vários grupos de pesquisa concluíram que o início da onda é o melhor momento para a aspiração, 
considerando-se a qualidade dos oócitos e o potencial de desenvolvimento embrionário (CASTILHO; ASSIS; GARCIA, 2007; HENDRIKSEN et al., 2004; MACHATKOVA et al., 2004).

A superioridade de produção de oócitos por raças zebuínas foi demonstrada por vários autores (NONATO JÚNIOR et al, 2004; RUBIN et al., 2004). Resultados obtidos anteriormente por nosso grupo (RUBIN et al., 2004) relataram uma média de 25 oócitos por sessão de aspiração folicular. As médias de oócitos recuperados por animal no presente trabalho foram menores do que as usualmente encontradas em bovinos da raça Nelore. Este fato pode ser explicado pelo baixo escore corporal (média 2,5, escala de 1 a 5), (LOWMAN; SCOTT; SOMERVILLE, 1976) dos animais durante este experimento. Segundo Dominguez (1995) e Lopez Ruiz et al., (1996), animais com baixa condição corporal apresentam uma redução na produção de oócitos de boa qualidade.

Neste trabalho 42,8\% (12/28) dos animais apresentaram corpo lúteo no momento da aspiração, destes, 35,7\% (10/28) pertenciam ao PAF, um número 5 vezes maior que o encontrado no $\mathrm{PCH}$ (2/28). Esta situação pode ser explicada pela ação coordenada da progesterona com o benzoato de estradiol no processo de liberação de prostaglandina (PGF2 $\alpha$ ) pelas células endometriais, que possui um importante papel na luteólise de bovinos (FANTINI et al., 2005; MIGUEZ et al., 2005). A ausência de CL no momento da aspiração folicular apresentou vários aspectos favoráveis. Pode-se destacar a maior facilidade de localização e punção dos folículos, bem como a redução da perfusão vascular, com menor captação de sangue.

Uma particularidade em relação aos protocolos para aspiração refere-se ao tipo de implante de progesterona utilizado. Os implantes intra-vaginais causam, em alguns animais, uma secreção vaginal mucóide. Embora esta secreção geralmente seja asséptica, decorrente de um processo irritativo, sua consistência viscosa pode dificultar o procedimento da aspiração folicular, por causar acúmulo de muco na extremidade do aparato de aspiração, com risco de entupir a agulha (SENEDA et al., 2006). Uma alternativa para isto são os implantes auriculares e/ ou a utilização de progesterona sintética injetável, sendo esta última de baixo custo quando comparada às citadas anteriormente e também pela praticidade de aplicação, não havendo o risco de perda do implante, nem a necessidade de retirá-lo ao fim do tratamento. No entanto, os resultados obtidos no presente trabalho sugerem a possibilidade de realização de protocolos pré-aspiração somente com progesterona injetável, devido ao curto período de início de uma nova onda de crescimento folicular após o protocolo. Em razão disto, consideramos a hipótese de não haver necessidade de um processo contínuo e prolongado de liberação de progesterona, qual ocorre nos implantes, sejam vaginais ou subcutâneos. Os resultados do presente estudo podem ser considerados uma etapa promissora para a confirmação desta hipótese.

Conclui-se que o protocolo controle hormonal $(\mathrm{PCH})$ foi mais eficaz na obtenção de oócitos, e proporcionou maior facilidade na manipulação dos ovários durante a aspiração em razão da redução significativa de corpos lúteos, bem como pela maior homogeneidade no tamanho dos folículos no momento da aspiração (maior o número de folículos $<5 \mathrm{~mm}$ ). Outro ponto a ser ressaltado no $\mathrm{PCH}$ é a praticidade do uso da progesterona injetável, podendo ser recomendada como alternativa de substituição aos implantes intra-vaginais ou auriculares de progestágeno.

\section{Agradecimentos}

Os autores agradecem a empresa Tecgene, em especial ao Prof. Joaquim Mansano Garcia. 


\section{Referências}

BARUSELLI, P. S.; SÁ FILHO, M. F.; MARTINS, C. M.; NASSER, L. F.; NOGUEIRA, M. F. G.; BARROS, C. M.; BÓ, G. A. Superovulation and embryo transfer in Bos indicus cattle. Theriogenology, Stoneham, v. 65, n. 1, p. 77-88, 2006.

BERFELT, D. R.; LIGHTFOOT, K. C.; ADAMS, G. P. Ovariam synchronization following ultrasound-guided transvaginal follicle ablation in heifers. Theriogenology, Stoneham, v. 42, n. 6, p. 895-907, 1994.

BLONDIN, P.; SIRARD, M. A. A oocyte and follicular morphology as determining characteristics for developmental competence in bovine oocytes. Molecular Reproduction and Development, New York, v. 41, n. 1, p. 54-62, 1995.

BÓ, G. A.; COLAZO, M. G.; MARTÍNEZ, M. F.; KASTELIC, J. P.; MAPLETOFT, R. J. Sincronizacion de la emergência de la onda folicular y la ovulación em animales tratados com progestagnos y diferentes ésteres de estradiol. In: SIMPÓSIO INTERNACIONAL DE REPRODUÇÃO ANIMAL APLICADA, 2., 2006, Londrina. Anais... Londrina: Ed. UEL, 2006. p. 71-84.

BÓ, G. A.; ADAMS, G. P.; PIERSON, R. A.; TRIBULO, H. E.; CACCIA, M.; MAPLETOFT, R. J. Follicular wave dynamics after estradiol-17 $\beta$ treatment of heifers with or without progestogen implant. Theriogenology, Stoneham, v. 41, n. 8, p. 1555-1569, 1994.

BOLS, P. E. J.; VANDENHEEDE, J. M. M.; VAN SOOM, A. Transvaginal ovum-pick-up (OPU) in the cow: a new disposable needle guindance system. Theriogenology, Stoneham, v. 43, n. 3, p. 677-687, 1995.

BOLS, P. E. J.; YSEBAERT, M. T.; LEIN, A.; CORYN, M.; VAN SOOM, A.; DE KRUIF, A. Effects of longterm treatment with bovine somatotropin on follicular dynamics and subsequent oocyte and blastocyst yield in an OPU-IVF program. Theriogenology, Stoneham, v. 49, n. 5, p. 983-995, 1998.

CASTILHO, C.; ASSIS, G. S.; GARCIA, J. M. Influência do diâmetro e da fase folicular sobre a competência in vitro de oócitos obtidos de novilhas da raça Nelore. Arquivo Brasileiro de Medicina Veterinária e Zootecnia, Belo Horizonte, v. 59, n. 2, p. 288-294, 2007.

CAVALIERI, J.; FARIN, P. W.; KINDER, J. E.; VAN CAMP, S. D.; WHITACRE, M. D.; WASHBURN, S. P.; BRITT, J. H. Ovarian follicular development following administration of progesterone or aspiration of ovarian follicles in Holstein cows. Theriogenology, Stoneham, v. 55 , n. 3, p. 805-821, 2001.
DE ROOVER, R.; GENICOT, G.; LEONARD, S.; BOLS, P.; DESSY, F. Ovum pick up and in vitro embryo production in cows superstimulated with an individually adapted superstimulation protocol. Animal Reproduction Science, Amsterdam, v. 86, n. 1, p. 13-25, 2005.

DOMINGUEZ, M. M. Effects of body condition, reproductive status and breed on follicular population and oocyte quality in cows. Theriogenology, Stoneham, v. 43, n. 8, p. 1405-1418, 1995.

FANTINI, D. A.; GOISSIS, M. D.; BAROS, F. R. O.; DIAS, L. O. P.; BRESSAN, F. F.; MIGUEZ, P. H. P.; BERTAN, C. M.; MARQUES, V. B.; ALMEIDA, A. B.; BINELLI, M. Efeitos de diferentes concentrações de progesterona durante o ciclo estral na liberação de prostaglandina PGF2 $\alpha$ induzida pelo 17 ß-estradiol em bovinos. In: REUNIÃO ANUAL DA SOCIEDADE BRASILEIRA DE TECNOLOGIA DE EMBRIÕES, 19., 2005, Angra dos Reis. Resumos... Porto Alegre: Ed. da UFRGS, 2005. p. 469.

GINTHER, O. J. Selection of the dominant follicle in cattle and horses. Animal Reproduction Science, Amsterdam, v. 60-61, p. 61-79, 2000.

GOODHAND, K. L.; WATT, R. G.; STAINES, M. E; HUTCHINSON, J. S. M.; BROADBENT, P. J. In vivo oocyte recovery and in vitro embryo production from bovine donors aspirated at different frequencies or following fsh treatment. Theriogenology, Stoneham, v. 51, n. 5, p. 951-961, 1999.

HENDRIKSEN, P. J. M.; STEENWEG, W. N. M.; HARKEMA, J. C.; MERTON, J. S.; BEVERS, M. M.; VOS, P. L. A. M., DIELEMAN, S. J. Effect of different stages of the follicular wave on in vitro developmental competence of bovine oocytes. Theriogenology, Stoneham, v. 61, n. 5, p. 909-920, 2004.

LOPEZ RUIZ, L.; ALVAREZ, N.; NUNEZ, I.; MONTES, I.; SOLANO, R.; FUENTES, D.; PEDROSO, R.; PALMA, G. A.; BREM, G. Effect of body condition on the developmental competence of IVM/IVF bovine oocytes. Theriogenology, Stoneham, v. 45, n. 1-2, p. 292, 1996. Abstract.

LOWMAN, B. G.; SCOTT, N.; SOMERVILLE, S. Condition scoring beef cattle. Edinburgh: East of Scotland College of Agriculture, 1976.

MACHATKOVA, M.; KRAUSOVA, K.; JOKESOVA, E.; TOMANEK, M. Developmental competence of bovine oocytes: effects of follicle size and the phase of follicular wave on in vitro embryo production. Theriogenology, Stoneham, v. 61, n. 2-3, p. 329-335, 2004. 
MIGUEZ, P. H. P.; CUNHA, P. M.; MARQUES, V. B.; BERTAN, C. M.; BINELLI, M. Combination of estradiol$17 \beta$ and progesterone is required for synthesis of PGF $2 \alpha$ in bovine endometrial explants. Animal Reproduction, Belo Horizonte, v. 2, n. 3, p. 172-177, 2005.

MINITAB INC. MINITAB: The student edition of Minitab statistical software adapted for education. 8. ed. New York: Addison-Wesley, 1992.

MORENO, D.; CUTAIA, L.; VILLATA, M. L.; ORTISI, F.; BÓ, G. A. Follicle wave emergence in beef cows treated with progesterone releasing devices, estradiol benzoate and progesterone. Theriogenology, Stoneham, v. 55, n. 1 , p. $408,2001$.

NONATO JÚNIOR, I.; RUFINO, F. A.; SANCHES, B. V.; PONTES, J. H. F.; UVO, S.; ERENO JÚNIOR, J. C.; SENEDA, M. M. Produção de embriões em vacas Nelore com a utilização associada de FIV e TE. In: REUNIÃO ANUAL DA SOCIEDADE BRASILEIRA DE TECNOLOGIA DE EMBRIÕES, 18., 2004, Barra Bonita. Resumos... Porto Alegre: UFRGS, 2004. v. 32. p. 95 .

PIETERSE, M. C.; VOS, P. L. A. M.; KRUIP, T. H. A. M.; WURTH, Y. A.; VAN BENEDEN, T. H. H.; WILlEMSE, A. H.; TAVERNE, M. A. M. Repeated transvaginal ultrasound-guided ovum pick-up in PMSGtreated cows. Theriogenology, Stoneham, v. 37, n. 1, p. 273, 1992. Abstract.

RUBIN, K. C. P.; RIGO, A. G.; SCHROEDER, R. V.; SILVA, R. C. P.; MARQUES, M. O.; SENEDA, M. M. Avaliação de uma bomba de infusão contínua como geradora de vácuo para obtenção in vivo de oócitos bovinos. In: REUNIÃO ANUAL DA SOCIEDADE BRASILEIRA DE TECNOLOGIA DE EMBRIÕES, 18. 2004, Barra Bonita. Resumos... Porto Alegre: UFRGS, 2004. v. 32. p. 121.

SENEDA, M. M.; ESPER, C. R.; ANDRADE, E. R.; BINELLI, M.; OLIVEIRA, J. A.; MAX, M. C.; GARCIA, J. M. Realtionship between follicle size after FSH treatment and efficiency of oocyte recovery. Animal Reproduction Science, Amsterdam, v. 2, n. 3, p. 178-182, 2005.
SENEDA, M. M.; ESPER, C. R.; GARCIA, J. M.; OLIVEIRA, J. A.; VANTINI, R. Relationship between follicle size and ultrasound-guided transvaginal oocyte recovery. Animal Reproduction Science, Amsterdam, v. 67, n. 1-2, p. 37-43, 2001.

SENEDA, M. M.; SANTOS, G. M. G.; SILVA, K. C. F.; SPEGIORIN, M. R.; BLASCHI, W.; PONTES, J. H. F. Situação atual da aspiração folicular e da fecundação in vitro. In: SIMPÓSIO INTERNACIONAL DE REPRODUÇÃO ANIMAL APLICADA, 2., 2006, Londrina. Anais... Londrina: Ed. da UEL, 2006. p. 172180.

SIRARD, M. A.; PICARD, L.; DERY, M.; COENEM, K.; BLONDIN, P. The time between FSH administration and ovarian aspiration influences the development of cattle oocytes. Theriogenology, Stoneham, v. 51, n. 4, p. 699-708, 1999.

TECHAKUMPHU, M.; PROMDIREG, A.; NACHIENGMAI, A.; PHUTIKANIT, N. Repeated oocyte pick up in prepubertal swamp buffalo (Bubalus bubalis) calves after FSH superstimulation. Theriogenology, Stoneham, v. 61, n. 9, p. 1705-1711, 2004.

TORRES-JÚNIOR, J. R. S.; PIRES, M. F. Á.; SÁ, W. F.; FERREIRA, A. M.; VIANNA, J. H. M.; CAMARGO, L. S. A.; RAMOS, A. A.; FOLHADELlA, I. M.; POLISSENI, J.; FREITAS, C.; CLEMENTE C. A. A.; SÁ FILHO, M. F.; SOUZA, A. H.; BARUSELLI, P. S. Efeito da codominância de folículos no momento da aspiração folicular sobre a recuperação e competência in vitro de oócitos Bos indicus. In: REUNIÃO ANULA DA SOCIEDADE BRASILEIRA DE TECNOLOGIA DE EMBRIÕES, 20., 2006, Araxá. Anais... Porto Alegrte: Ed. da Universidade Federal do Rio Grande do Sul, 2006. p. 473. Resumo.

WALTON, J. S.; CHRISTIE, K. A.; STUBBINGS, R. B. Evaluation of frequency of ultrasonically guided follicle aspiration on bovine ovarian dynamics. Theriogenology, Stoneham, v. 9, n. 1, p. 6, 1993. Abstract. 
